\title{
Indicadores de estresse e qualidade da carne em frangos abatidos pelo método "Halal"
}

\section{Indicators of stress and quality meat in broilers slaughtered by the "Halal"}

\author{
Ana Maria Bridi ${ }^{1 *}$; Nilva Aparecida Nicolao Fonseca ${ }^{2}$; Caio Abércio da Silva ${ }^{1}$; \\ Mara Regina Stipp Balarin 3 ; Karina Keller Marques da Costa Flaiban ${ }^{3}$; Camila \\ Costantino ${ }^{4}$; Marina Avena Tarsitano ${ }^{4}$; Thales de Almeida Bitencourt Cardoso 5
}

\section{Resumo}

Este trabalho teve como objetivo avaliar o estresse e a qualidade da carcaça e da carne de frangos abatidos pelo método "Halal". Foram avaliadas 120 carcaças, 60 frangos abatidos pelo método tradicional e 60 frangos abatidos pelo método "Halal", com peso médio de 3,8 $\mathrm{kg}$ de peso vivo e idade de 49 dias. No momento da sangria foi coletado sangue para análise dos níveis de creatina fosfoquinase, lactato e hemograma. Após o abate foi verificado nas carcaças a presença de hematomas, contusões, hemorragias musculares e fraturas. No músculo peitoral (Pectoralis major) foi mensurado o $\mathrm{pH}$ inicial e final, a cor (CIELAB), a perda de água por gotejamento e por pressão e a maciez (Força de cisalhamento). Os níveis de lactato foram mais elevados e de monócitos mais baixos nos frangos abatidos pelo método "Halal", indicando maior nível de estresse, embora a porcentagem de hematócrito tenha sido maior para o abate tradicional. Animais abatidos pelo método "Halal" apresentaram lesões de maior tamanho nas asas e carnes com menores valores de $\mathrm{pH}$ inicial e final, maior luminosidade $\left(\mathrm{L}^{*}\right)$ e maior índice de vermelho (a*). Foi observado maior frequência de carcaças PSE (cor pálida, textura mole, exsudativa) nos animais abatidos pelo método "Halal". Conclui-se que o método de abate Halal para frangos, aumenta o estresse assim como o tamanho das lesões das carcaças e maior frequência de carcaças PSE, diminuindo a qualidade da carne de frango.

Palavras-chave: Abate humanitário, abate tradicional, insensibilização elétrica, Halal, PSE

\begin{abstract}
This research was aimed to evaluate the stress as well as carcass and meat quality of broilers slaughtered by the "Halal" method. 120 carcasses were evaluated, 60 broilers were slaughtered by the traditional method and 60 broilers by the "Halal" method, with live weight of $3.8 \mathrm{~kg}$ and 49 days old. Exsanguination blood samples were collected to measure the levels of creatine phosphokinase, lactic acid and hemogram. After the slaughter each carcass had been evaluated to verify the presence of bruises, contusions, muscle bleeding, and fractures. Initial and final $\mathrm{pH}$, color (CIELAB), drip (PAG) and pressing water loss (PAP)
\end{abstract}

${ }^{1}$ Profs. Drs. do Dept ${ }^{0}$ de Zootecnia. Universidade Estadual de Londrina, UEL, Londrina, PR. E-mail: ambridi@uel.br; casilva@ uel.br

${ }^{2}$ Prof $^{\mathrm{a}}$. Dr ${ }^{\mathrm{a}}$ Aposentada do Dept ${ }^{\mathrm{o}}$ de Zootecnia, UEL, Londrina, PR. E-mail: nilva@uel.br

${ }^{3}$ Profs. Drs. do Dept ${ }^{0}$ de Medicina Veterinária Preventiva. Universidade Estadual de Londrina, UEL, Londrina, PR. E-mail: maravet@uel.br; kkflaiban@uel.br

${ }^{4}$ Discente(s) de Doutorado do Programa de Pós-Graduação em Ciência Animal, UEL, Londrina, PR. E-mail: caconstantino@ hotmail.com; marina_avena@yahoo.com.br

${ }^{5}$ Discente de Mestrado do Programa de Pós-Graduação em Ciência Animal, UEL, Londrina, PR. E-mail: thales.cardoso@, zootecnista.com.br

* Autor para correspondência 
and tenderness (shear force-FC) were measured in "Pectoralis major" muscle. The lactic acid levels were higher and lower in monocytes for the broilers slaughtered using the "Halal" method, indicating higher stress levels, while the percentage of hematocrit was great to the traditional slaughter. Animals slaughtered by the "Halal" method had wing bruises with bigger size and meat with lower $\mathrm{pH}$ values the initial and final, higher lightness ( $\left.\mathrm{L}^{*}\right)$ and higher redness ( $\left.\mathrm{a}^{*}\right)$. It was observed higher frequency of PSE carcass (pale, soft, exsudative) slaughtered by method "Halal". It is concluded that the "Halal" method of slaughter for chickens, stress increases and the size of the lesions of carcasses and higher frequency of PSE carcasses, reducing the quality of chicken meat.

Key words: Humane slaughter, conventional slaughter, electrical stunning, Halal, PSE

\section{Introdução}

O Islamismo impõe regras à alimentação de seus adeptos. Os alimentos permitidos são chamados de "Halal", palavra que significa "permitido, reservado, autorizado, aprovado, legal, lícito" (GOMIDE; RAMOS; FONTES, 2006).

Segundo Cibal Halal (2005) o processo de certificação "Halal" teve início no Brasil em 1997. No abate "Halal" o animal deve ser sacrificado fazendo um corte no pescoço em forma de meialua. Deve-se seccionar simultaneamente a jugular, a traquéia, as artérias carótidas e o esôfago, fazendo com que o sangue seja totalmente e espontaneamente extraído da carcaça.

O Brasil é o maior exportador mundial de carne de frango, sendo que mais de $25 \%$ dos animais são abatidos pelo método "Halal". O abate "Halal" atende a comunidade islâmica local e exporta para o Oriente Médio e outros países com população Mulçumana na Europa, Ásia e África, sendo a Arábia Saudita o maior comprador (SINDIAVIAPAR, 2010).

A Instrução Normativa No 3 de 17 de janeiro de 2000 do Ministério da Agricultura, Pecuária e Abastecimento (BRASIL, 2000), que regulamenta os Métodos de Insensibilização para o Abate Humanitário, obriga a insensibilização dos animais destinados ao abate. A mesma legislação, entretanto, permite o sacrifício dos animais de acordo com preceitos religiosos.

O método de insensibilização mais usado em aves é a eletronarcose, sendo aprovada pelas legislações mundiais de abate humanitário. O processo consiste na completa e instantânea inconsciência do animal, pela passagem de corrente elétrica pelo cérebro, fazendo com que ele possa ser abatido sem sofrer dor e angústia, reduzindo a resposta ao estresse no momento do abate (GOMIDE; RAMOS; FONTES, 2006) e facilitando, pela imobilização, o corte automático do pescoço.

No abate "Halal" a insensibilização geralmente não é usada porque provoca um decréscimo na perda de sangue pela carcaça, um aspecto que contraria a crença islâmica. Por isso, o abate "Halal" tem sido questionado pelos defensores dos direitos dos animais que alegam que o sacrifício sem insensibilização pode causar dor e sofrimento.

O bem-estar dos animais pode ser avaliado através das mudanças fisiológicas e comportamentais provocadas pelo estresse. Os principais indicadores plasmáticos do estresse são o lactato e o cortisol (DALLA COSTA et al., 2008).

Nos animais estressados pouco tempo antes do abate, o pH final da carne é atingido antes de completar uma hora post mortem devido à alta velocidade da glicólise. Este fenômeno pode levar à ocorrência da anomalia PSE (cor pálida, textura mole, exsudativa). As carnes PSE apresentam coloração mais clara porque a baixa capacidade de retenção de água faz com que pigmentos, como a mioglobina, sejam carreados junto com a água para fora das células. Também, o acúmulo de água nos espaços extracelulares resulta em menor absorção e maior refração da luz (LAWRIE, 2005).

Desta forma, - este trabalho teve como objetivo avaliar o estresse e a qualidade da carcaça e da carne de frangos abatidos pelo método "Halal". 


\section{Material e Métodos}

O experimento foi desenvolvido na Fazenda Escola e as análises de qualidade da carne foram realizadas no Laboratório de Análises de Produtos de Origem Animal do Departamento de Zootecnia da Universidade Estadual de Londrina. Foram avaliadas 120 carcaças, sendo 60 de frangos abatidos pelo método tradicional (T1), insensibilizados por choque elétrico e 60 frangos abatidos pelo método "Halal" (T2). O delineamento experimental utilizado foi completamente casualizado. Cada frango foi considerado uma unidade experimental.

$\mathrm{O}$ processo de abate tradicional consistiu na insensibilização elétrica $(50 \mathrm{~V}, 1000 \mathrm{~Hz}, 1 \mathrm{~A})$ (Fluxo, modelo FX 20, Chapecó, SC), seguido da sangria (BRASIL, 2000). Os frangos abatidos pelo método "Halal" não foram insensibilizados, sendo sacrificados através do corte direto dos grandes vasos do pescoço e esôfago (degola sangrenta) (GOMIDE; RAMOS; FONTES, 2006)

No momento da sangria foram coletados três amostras de $5 \mathrm{~mL}$ de sangue de cada animal para análise dos níveis de creatina fosfoquinase, lactato e determinação do hemograma. No hemograma foram quantificados os valores de hematócritos, hemoglobina, hemácias, leucócitos, heterófilos, proteína plasmática total, eosinófilos, monócitos e linfócitos conforme as metodologias descritas a seguir.

A creatina fosfoquinase foi determinada no soro utilizando o método enzimático - UV (Kovalent, São Gonçalo, RJ), por meio da metodologia otimizada de acordo com German Society for Clinical Chemistry (1977). O lactato foi quantificado no plasma fluoretado sanguíneo, utilizando o método enzimático - UV (Kit enzimático Kovalent, São Gonçalo, RJ) segundo Thomas (1998).

A determinação dos hematócritos foi realizada por meio do método de microhematócrito, utilizando-se tubo capilar centrifugado a 11.360 $\mathrm{G}$ por 5 minutos em microcentrífuga (EVILAB, modelo 024, Londrina, PR). A contagem de hemácias e de leucócitos foi realizada em hemocitômetro em amostra de sangue diluída a 1:200, em corante de Natt e Herrick's. A concentração de hemoglobina foi determinada pelo método da cianometaemoglobina (Kit enzimático Bioclin, São Paulo, SP) com leitura em espectrofotômetro (modelo BIO2000, BIOPLUS, Barueri, SP). A contagem diferencial leucocitária foi realizada em esfregaço sanguíneo, fixado com álcool metílico (Metanol) e submetido à coloração com o corante rápido para hematologia (Panótipo rápido).

Após passarem pelo resfriamento e gotejamento, as carcaças foram analisadas individualmente para a verificação de presença de hematomas, contusões, hemorragias musculares e fraturas. Foram anotados a presença, o local (perna, coxa, dorso, peito e asa) e o tamanho das lesões, com auxílio de padrões fotográficos, segundo Huallanco (2004).

De cada carcaça foi retirada uma amostra do músculo do peito totalizando 60 amostras para o método de abate tradicional e 60 amostras para o método de abate "Halal". Estas foram identificadas e acondicionadas em caixas de isopor e transportadas para o laboratório para serem submetidas às análises de cor e $\mathrm{pH}$.

$\mathrm{O} \mathrm{pH}$ da carne foi aferido no músculo Pectoralis major 1 hora ( $\mathrm{pH}$ inicial) e 24 horas após o abate sob refrigeração de $0 \pm 2{ }^{\circ} \mathrm{C}$ ( $\mathrm{pH}$ final), sendo uma leitura em cada tempo, utilizando um potenciômetro portátil com eletrodo de inserção (Testo, modelo 205, Lenzkirch, Germany).

A cor foi analisada nas amostras após 30 minutos de exposição ao oxigênio, para reação da mioglobina com o oxigênio atmosférico, sendo realizadas três leituras por amostra por meio do aparelho colorímetro portátil com iluminante $\mathrm{C}$ e ângulo de observação de $8^{\circ}$ (Konica Minolta, Color reader CR10, Mahwah, EUA) na superfície ventral do músculo Pectoralis major. Os componentes luminosidade $\left(\mathrm{L}^{*}\right)$, índice de vermelho $\left(\mathrm{a}^{*}\right)$ e índice de amarelo $\left(\mathrm{b}^{*}\right)$ foram expressos no sistema de cor CIELAB. Com essas médias calculou-se 
a saturação (c*) e o ângulo de tonalidade (h*) da carne (MINOLTA, 1998).

Os valores de $\mathrm{L}^{*}$ fornecidos pelo colorímetro e os valores de $\mathrm{pH}$ foram utilizados para classificar as carnes em normais, PSE (cor pálida, textura mole e exsudativa,) e DFD (cor escura, textura firme e que retém muita água). As carnes foram classificadas como PSE quando apresentaram valores de $\mathrm{pH}$ inicial inferiores a 5,8 e valor $\mathrm{L}^{*}$ maior que $53 \mathrm{e}$ como DFD quando apresentaram $\mathrm{pH}$ final maior que 6,0 e valor L* menor que 44 (OLIVIO, 2006).

A perda de água por gotejamento foi analisada segundo a técnica descrita por Boccard et al. (1981). As amostras do músculo Pectoralis major foram pesadas em balança semi analítica e suspensas por um gancho de aço galvanizado inoxidável em forma de "S" para que uma extremidade sustentasse a carne e a outra ficasse presa nas grades da geladeira. As amostras foram colocadas dentro de uma camada dupla de saco de polietileno, estes foram inflados e fechados com barbante, mantidos por 48 horas sob refrigeração a $4^{\circ} \mathrm{C}$. Posteriormente a este período, as amostras foram pesadas novamente para ser calculada a perda de água por gotejamento. A perda de água por pressão foi realizada seguindo a técnica descrita por Bridi e Silva (2009). Foram pesados dois gramas da amostra do músculo do peito em balança semi-analítica. A amostra foi posicionada entre dois papéis filtro (Whatman n.1) e pressionouse a amostra entre duas placas de acrílico com um peso de $10 \mathrm{~kg}$ por cinco minutos. Após a prensagem a amostra foi pesada novamente para ser calculada a perda de água.

Para avaliar a maciez da carne, utilizaramse as amostras das análises de perda de líquido por descongelamento e cocção. Todo a porção do músculo Pectoralis major foi pesada congelada e pesada novamente após 24 horas de descongelamento a $4 \pm 2{ }^{\circ} \mathrm{C}$ (perda de líquido por descongelamento). Posteriormente, e foram assadas em forno elétrico pré-aquecido por 20 minutos a $180^{\circ} \mathrm{C}$ até atingirem a temperatura interna de $72^{\circ} \mathrm{C}$. Após a cocção, as amostras foram armazenadas por 24 horas a $4 \pm 2{ }^{\circ} \mathrm{C}$ e pesadas novamente (perda de líquido na cocção) (BRIDI; SILVA, 2009). Foram retiradas, de cada amostra, seis sub-amostras cilíndricas de $2,5 \mathrm{~cm}$ de comprimento e $1,27 \mathrm{~cm}$ de diâmetro, utilizando-se um amostrador de aço de forma cilíndrica. A força de cisalhamento foi medida perpendicularmente à orientação das fibras musculares com a lâmina Warner-Bratzler adaptada ao texturômetro (Modelo TA-XT2i, Stable Mycro Systems LTDA., Goldalming, UK) (WHIPPLE et al., 1990). As velocidades utilizadas foram de $5 \mathrm{~mm} / \mathrm{s}$ no pré e pós-teste e de $2 \mathrm{~mm} / \mathrm{s}$ no teste.

Os dados foram submetidos ao Teste F utilizando o programa estatístico SAEG (1997). Para as variáveis frequência de lesões nas asas e de carnes PSE, em que os resultados foram expressos em porcentagem, foi utilizado o teste não paramétrico do Qui-quadrado. Foram também realizadas análises de correlação de Pearson entre as variáveis relacionadas à qualidade da carne.

\section{Resultados e Discussão}

Os níveis de ácido lático diferiram significativamente entre os dois tratamentos, com maior valor observado para o abate "Halal" (Tabela 1). O lactato é produto da glicólise anaeróbica celular. Níveis elevados de lactato no sangue estão associados ao aumento de metabolismo provocado pelo estresse (BERTOLONI et al., 2006). 
Tabela 1. Teores de ácido lático e creatina fosfoquinase no sangue de frangos abatidos por diferentes métodos ${ }^{(1)}$.

\begin{tabular}{lcc}
\hline \multicolumn{1}{c}{ Variável } & \multicolumn{2}{c}{ Métodos de abate } \\
\cline { 2 - 3 } & "Halal" & Tradicional \\
\hline Lactato $(\mathrm{mg} / \mathrm{dL})$ & $36,48 \pm 11,34^{*}$ & $27,26 \pm 7,68^{*}$ \\
Creatina fosfoquinase (U/L) & $18.961,91 \pm 8267,23^{\text {ns }}$ & $17.705,10 \pm 8659,33^{\text {ns }}$ \\
\hline
\end{tabular}

(1) ns, Não significativo. *Diferenças significativas a $5 \%$ de probabilidade de acordo com teste $\mathrm{F}$.

Fonte: Elaboração dos autores.

Com relação à creatina fosfoquinase não foram observadas diferenças significativas entre os tratamentos. A creatina fosfoquinase é uma enzima envolvida na transformação reversível da ADP (adenosina difosfato) e fosfocreatina em ATP (adenosina trifosfato), que assegura o suplemento de energia intracelular. Sob uma situação de estresse, a liberação de cortisona nas aves provoca a aceleração do metabolismo muscular, que resulta no aumento da atividade de creatina fosfoquinase (MITCHELL; SANDERCOCK, 1995; LIN; DECUYPERE; BUYSE, 2004).
As médias de hematócrito foram estatisticamente diferentes entre si, com o valor maior para o abate precedido de eletronarcose (Tabela 2). Em ambos os tratamentos, os níveis de hematócrito (ou volume globular) encontraram-se na faixa de normalidade, que em frangos situa-se entre 22 e $35 \%$. Esperava-se uma elevação nos níveis de hematócrito em situação de estresse, devido à contração do baço, que libera hemácias extras na corrente sanguínea (BATISTA et al., 2009).

Tabela 2. Componentes do hemograma de frangos abatidos por diferentes métodos ${ }^{(1)}$.

\begin{tabular}{lrr}
\hline \multirow{2}{*}{ Parâmetros avaliados } & \multicolumn{2}{c}{ Métodos de abate } \\
\cline { 2 - 3 } & \multicolumn{1}{c}{ "Halal" } & \multicolumn{1}{c}{ Tradicional } \\
\hline Hematócrito $(\%)$ & $30,00 \pm 1,81^{*}$ & $31,13 \pm 1,06^{*}$ \\
Hemoglobina $(\mathrm{g} / \mathrm{dL})$ & $7,60 \pm 1,37^{\text {ns }}$ & $7,64 \pm 0,60^{\text {ns }}$ \\
Hemácias $\left(\mathrm{M} / \mathrm{mm}^{3}\right)$ & $2,31 \pm 0,17^{\text {ns }}$ & $2,33 \pm 0,11^{\text {ns }}$ \\
Leucócitos $\left(\mathrm{M} / \mathrm{mm}^{3}\right)$ & $6050,0 \pm 2172,83^{\text {ns }}$ & $6556,0 \pm 2363,92^{\text {ns }}$ \\
Proteínas Plasmáticas Totais (g/dL) & $3,68 \pm 0,20^{\text {ns }}$ & $3,72 \pm 0,34^{\text {ns }}$ \\
Heterófilos $(\%)$ & $2447,50 \pm 1350,63^{\text {ns }}$ & $2494,37 \pm 2044,57^{\text {ns }}$ \\
Eosinófilos $(\%)$ & $2,67 \pm 2,10^{\text {ns }}$ & $2,33 \pm 2,41^{\text {ns }}$ \\
Linfócitos $(\%)$ & $57,50 \pm 14,12^{\text {ns }}$ & $58,67 \pm 19,47^{\text {ns }}$ \\
Monócitos $(\%)$ & $0,33 \pm 0,78^{*}$ & $2,75 \pm 1,29^{*}$ \\
Relação heterófilo/linfócito & $42,56 \pm 3,48^{\text {ns }}$ & $42,51 \pm 5,12^{\text {ns }}$ \\
\hline
\end{tabular}

(1) ns, Não significativo. * Diferenças significativas a 5\% de probabilidade de acordo com teste F.

Fonte: Elaboração dos autores.

A proporção de eosinófilos não foi afetada, entretanto, foi observada redução no número de monócitos no sangue dos frangos abatidos pelo método "Halal". Segundo Altan et al. (2000) a exposição ao estresse agudo resulta no decréscimo da proporção de monócitos no sangue.
A avaliação da relação heterófilos/linfócitos é usada como um índice de estresse crônico em aves, ou seja, estresse de longa duração (GROSS; SIEGEL, 1983; SCOPE et al., 2002), uma vez que o estresse crônico provoca um aumento nos heterófilos e/ou redução no número de linfócitos. Os frangos 
abatidos pelo método "Halal" ou insensibilizados não apresentaram diferença estatística para este parâmetro, indicando que no estresse agudo não há tempo suficiente para que ocorram mudanças nos valores de heterófilos e linfócitos.

Em relação às lesões nas carcaças, somente foram verificados danos na região das asas. A frequência de lesões e a dimensão das lesões nas asas foram influenciadas pelo método de abate. No método "Halal" houve maior frequência de lesões e de maior tamanho (Tabela 3). Diante da ausência da eletronarcose no método "Halal", no momento do corte dos grandes vasos, os animais apresentaram sensibilidade, sofrendo estresse e se debatendo mais durante o processo de sangria, resultando em maior número de lesões e de maior dimensão.

Tabela 3. Parâmetros físicos e de qualidade da carne de frangos abatidos por diferentes métodos ${ }^{(1)}$.

\begin{tabular}{lrr}
\hline \multirow{2}{*}{ Parâmetros avaliados } & \multicolumn{2}{c}{ Método de abate } \\
\cline { 2 - 3 } & \multicolumn{1}{c}{ "Halal" } & Tradicional \\
\hline Peso abate $(\mathrm{kg})$ & $3,81 \pm 0,16^{\mathrm{ns}}$ & $3,77 \pm 0,21^{\mathrm{ns}}$ \\
Frequência de lesão nas asas $\left(\mathrm{cm}^{2}\right)$ & $38,33^{* *}$ & $33,29^{* *}$ \\
Tamanho da lesão nas asas $\left(\mathrm{cm}^{2}\right)$ & $4,42 \pm 1,45^{*}$ & $2,68 \pm 0,78^{*}$ \\
pH inicial & $5,88 \pm 0,11^{*}$ & $6,39 \pm 0,13^{*}$ \\
pH final & $5,86 \pm 0,09^{*}$ & $5,93 \pm 0,11^{*}$ \\
PAG (\%) & $3,46 \pm 1,75^{\mathrm{ns}}$ & $3,81 \pm 2,11^{\mathrm{ns}}$ \\
PAP (\%) & $31,03 \pm 3,41^{\mathrm{ns}}$ & $30,67 \pm 3,33^{\mathrm{ns}}$ \\
L $^{*}$ & $54,34 \pm 1,49^{*}$ & $52,85 \pm 2,91^{*}$ \\
a $^{*}$ & $0,54 \pm 0,90^{*}$ & $-0,47 \pm 0,51^{*}$ \\
$\mathrm{~b}^{*}$ & $12,13 \pm 1,30^{\mathrm{ns}}$ & $11,59 \pm 1,23^{\mathrm{ns}}$ \\
Croma & $12,17 \pm 1,32^{\mathrm{ns}}$ & $11,61 \pm 1,23^{\mathrm{ns}}$ \\
Tonalidade (graus) & $87,72 \pm 4,23^{*}$ & $92,34 \pm 2,45^{*}$ \\
Maciez (kgf) & $3,61 \pm 0,94^{\mathrm{ns}}$ & $4,66 \pm 1,38^{\mathrm{ns}}$ \\
PSE \% & $23,33^{* *}$ & $0^{* *}$ \\
DFD \% & $0^{\mathrm{ns}}$ & $0^{\mathrm{ns}}$ \\
\hline
\end{tabular}

(1) ns, Não significativo. *Diferenças significativas a $5 \%$ de probabilidade de acordo com teste F. ** Diferenças significativas a 5\% de probabilidade para o teste do Qui-quadrado. PAG: perda de água por gotejamento; PAP: perda de água por pressão.

Fonte: Elaboração dos autores.

Os valores de $\mathrm{pH}$ inicial e final foram influenciados pelo método de abate, com as carcaças abatidas pelo método "Halal" apresentando valores inferiores (Tabela 3). A glicólise anaeróbica resulta na formação e acúmulo de lactato no músculo, que reduz o $\mathrm{pH}$ da carne (LAWRIE, 2005). Em aves, o estresse pré-abate provoca liberação dos hormônios catecolaminas e glicocorticóides. Esses hormônios elevam o metabolismo dos animais, fazendo com que a glicólise anaeróbica ocorra a uma velocidade muito acima do normal, resultando em valores mais baixos de pH (ALI; KANG; JOO, 2008). Os menores valores de $\mathrm{pH}$ inicial e final observados no peito dos frangos abatidos pelo método "Halal", não resultaram em maiores perdas de água por gotejamento ou pressão.

A luminosidade da carne, indicada pelo valor de $\mathrm{L}^{*}$, foi maior nos animais abatidos pelo método "Halal". A luminosidade apresenta correlação negativa com o valor de $\mathrm{pH}$ da carne. Assim, era de se esperar que animais que apresentassem menor valor de $\mathrm{pH}$ apresentassem maior valor de $\mathrm{L}^{*}$, ou seja, carne de cor mais clara.

O valor de $a^{*}$ foi superior na carne de peito de frangos abatidos pelo método "Halal", indicando 
maior participação da cor vermelha, fato que sugere uma pior sangria para esse tratamento. Entretanto, Northcutt, Buhr e Young (1998) não verificaram diferença na cor da carne de peitos de perus insensibilizados ou não por eletronarcose antes do abate. Já Roça et al. (2001) encontram melhor eficiência de sangria em bovinos abatidos pelo método Kasher quando comparado com os insensibilizados por pistola pneumática.

Os valores de $b^{*}$ e croma $\left(c^{*}\right)$ não foram influenciados pelo método de abate. $\mathrm{O}$ ângulo de tonalidade $\left(\mathrm{h}^{\circ}\right)$ foi influenciado pelo método de abate, sendo que o abate "Halal" proporcionou menor valor de $h^{\circ}$. Isto era esperado pois, o valor $a^{*}$ foi alterado pelo mesmo método de abate.

A frequência de carcaças PSE foi de 23,33\% para os frangos abatidos no método "Halal", contra zero por cento no método tradicional com insensibilização por eletronarcose. Nos animais estressados, o metabolismo post mortem ocorre de forma acelerada provocando rápida degradação do glicogênio e acúmulo do ácido láctico. Neste caso, o pH final é atingido antes de uma hora após o abate. $\mathrm{O} \mathrm{pH}$ baixo, enquanto a temperatura da carcaça ainda está alta, leva à desnaturação proteica e ao aparecimento de carne PSE (LAWRIE, 2005). Não foi verificada nenhuma incidência de carne DFD. O DFD é uma anomalia mais frequente quando os animais passam por estresse prolongado antes do abate, exaurindo suas reservas de glicogênio.

Segundo a análise de correlação de Pearson (Tabela 4), os valores de $\mathrm{pH}$ inicial e $\mathrm{pH}$ final apresentaram correlação positiva $(\mathrm{P}<0,05)$ com a tonalidade da carne e negativa com o valor de $\mathrm{L}^{*}$ e o croma, indicando que quanto menor o valor do $\mathrm{pH}$ da carne de frango, maior o valor de $\mathrm{L}^{*}$, ou seja, uma carne mais clara, com maior índice de saturação (croma) e menor tonalidade. O valor de L* apresentou correlação positiva com a perda de água por pressão e o croma, ou seja, as carnes mais claras (maior valor de $\mathrm{L}^{*}$ ) apresentaram maior perda de água e maior saturação da cor.

Tabela 4. Análise de correlação de Pearson entre as características de qualidade da carne.

\begin{tabular}{lccccc}
\hline & $\mathrm{L}^{*}$ & PAG $(\%)$ & PAP $(\%)$ & Croma & $\begin{array}{c}\text { Tonalidade } \\
\text { (graus) }\end{array}$ \\
\hline pH inicial & $-0,326$ & - & - & $-0,299$ & 0,555 \\
pH final & $-0,618$ & 0,229 & $-0,258$ & $-0,409$ & 0,239 \\
$\mathrm{~L}^{*}$ & - & - & 0,459 & 0,562 & - \\
\hline
\end{tabular}

PAG: perda de água por gotejamento; PAP: perda de água por pressão. Nível de significância de 5\%

Fonte: Elaboração dos autores.

\section{Conclusão}

Nas condições do presente experimento, o método de abate "Halal" para frangos elevou o estado de estresse resultando no aumento da concentração de lactato e maior frequência de carcaças lesionadas e com carne PSE.

\section{Agradecimentos}

Ao $\mathrm{CNPq}$ pelo apoio financeiro para o desenvolvimento da pesquisa. 


\section{Referências}

ALI, S.; KANG, G.-H.; JOO, S. T. A review: influences of pre-slaughter stress on poultry meat quality.AsianAustralasian Journal of Animal Sciences, Seoul, v. 21, n. 6, p. 912-916, 2008.

ALTAN, O.; ALTAN, A.; ÇABUK, M.; BAYRAKTAR, H. Effects of heat stress on some blood parameters in broilers. Turkish Journal of Veterinary and Animal Sciences, Ankara, v. 24, n. 2, p. 145-148, 2000.

BARBUT, S.; SOSNICKI, A. A.; LONERGAN, S. M.; KNAPP, T.; CIOBANU, D. C.; GATCLIFFE, L. J.; HUFF-LONERGAN, E.; WILSON, E. W. Progress in reducing the pale, soft and exudative (PSE) problem in pork and poultry meat. Meat Science, Barking, v. 79, n. 1, p. 46-63, 2008.

BATISTA, J. S.; BEZERRA, F. S. B.; AGRA, E. G. D.; CALADO, E. B.; GODÓI, R. M.; RODRIGUES, C. M. F.; NUNES, F. C. R.; BLANCO, B. S. Efeitos da contenção física e química sobre os parâmetros indicadores de estresse em catetos (tayassu tajacu). Acta Veterinaria Brasilica, Mossoró, v. 3, n. 2, p. 92-97, 2009.

BERTOLONI, W.; SILVEIRA, E. T. F.; LUDTKE, C. B.; ANDRADE, J. C. Avaliação de diferentes híbridos suínos submetidos à insensibilização elétrica e gasosa (Co2). Parte 1 - mensuração de indicadores sanguíneos de estresse. Ciência e Tecnologia de Alimentos, Campinas, v. 26, n. 3 , p. $564-570,2006$.

BOCCARD, R.; BUCHTER, L.; CASTEELS, E.; COSENTINO, E.; DRANSFIELD, E.; HOOD, D. E.; JOSEPH, R. L.; MACDOUGALL, D. B.; RHODES, D. N.; SCHÖN, I.; TINBERGEN, B. J.; TOURAILLE, C. Procedures for measuring meat quality characteristics in beef production experiments. Livestock Production Science, Amsterdam, v. 8, n. 5, p. 385-397, 1981.

BRASIL. Ministério da Agricultura, Pecuária e do Abastecimento. Divisão de Normas Técnicas. Instrução normativa $\mathrm{n}^{\circ} 3$, de 17 de janeiro de 2000. Aprova o regulamento técnico de métodos de insensibilização para o abate humanitário de animais de açougue. Diário Oficial [da] República Federativa do Brasil, Brasília, 24 jan. 2000. Seção 1, p. 14-16.

BRIDI, A. M.; SILVA, C. A. Avaliação da carne suína. 2. ed. Londrina: Midiograf, 2009. 120 p.

CIBAL HALAL. O que é Halal. 2005. Disponível em: $<$ http://www.cibalHalal.com.br/>. Acesso em: 12 ago. 2010 .

DALLA COSTA, O. A.; LUDKE, J. V.; COSTA; M. J. R. P.; FAUCITANO, L.; COLDEBELLA, A.; KICH, J. D.; PELOSO, J. V.; ROZA, D. D. Tempo de jejum na granja sobre o perfil hormonal e os parâmetros fisiológicos em suínos de abate pesados. Ciência Rural, Santa Maria, v. 38, n. 8, p. 2300-2306, 2008.

GERMAN SOCIETY FOR CLINICAL CHEMISTRY. Standardization of methods for the estimation of enzyme activities in biological fluids: Standard method for the determination of creatine Kinase activity. Journal of Clinical Chemistry and Clinical Biochemistry, Berlin, v. 15, p. 255-260, 1977.

GOMIDE, L. A. M.; RAMOS, E. M.; FONTES, P. R. Tecnologia de abate e tipificação de carcaças. Viçosa: UFV, 2006. 599 p.

GROSS, W. B.; SIEGEL, H. S. Evaluation of the heterophil/lymphocyte ratio as a measure of stress in chickens. Avian Diseases, Jacksonville, v. 27, n. 4, p. 972-979, 1983.

HUALLANCO, M. B. A. Aplicação de um sistema de classificação de carcaças e cortes de frango criados no sistema alternativo. 2004. Dissertação (Mestrado em Ciência e Tecnologia de Alimentos) - Escola Superior de Agricultura Luiz de Queiroz, Piracicaba.

LAWRIE, R. A. Ciência da carne. Porto Alegre: Artmed, 2005. 384 p.

LIN, H.; DECUYPERE, E.; BUYSE, J. Oxidative stress induced by corticosterone administration in broiler chickens (Gallus gallus domesticus) 1. Chronic exposure. Comparative Biochemistry and Physiology - Part B: Biochemistry \& Molecular Biology, New York, v. 139, n. 4, p.737-744, 2004.

MINOLTA Co. Precise color communication: color control from perception to instrumentation. Japan: Minolta, 1998. 59 p.

MITCHELL, M. A.; SANDERCOCK, D. A. Creatine kinase isoenzyme profiles in the plasma of the domestic fowls (Gallus domesticus): effects of acute heat stress. Research in Veterinary Science, Oxford, v. 59, n. 1, p. 30-34, 1995.

NORTHCUTT, J. K.; BUHR, R. J.; YOUNG, L. L. Influence of preslaughter stunning on turkey breast muscle quality. Poultry Science, Ithaka, v. 77, n. 3, p. 487-492, 1998.

OLIVIO, R. O mundo do frango: cadeia produtiva da carne de frango. Criciúma: Ed. do Autor, 2006. 680 p.

ROÇA, R. de O.; PADOVANI, C. R.; FILIPI, M. C. de; SCHWACH, E.; UEMI, A.; SHINKAI, R. T.; BIONDI, G. F. Efeitos dos métodos de abate de bovinos na eficiência da sangria. Ciência e Tecnologia de Alimentos, Campinas, v. 21, n. 2, p. 244-248, 2001. 
UNIVERSIDADE FEDERAL DE VIÇOSA - UFV. SAEG - Sistema de análises estatísticas e genéticas. Versão 7.1. Viçosa, MG: 1997. 157 p. (Manual do usuário).

SCOPE, A.; FILIP, T.; GABLER, C.; RESCH, F. The influence of stress from transport and handling on hematologic and clinical chemistry blood parameters of racing pigeons (Columba livia domestica). Avian Diseases, Jacksonville, v. 46, n. 1, p. 224-229, 2002.

SINDICATO DAS INDÚSTRIAS DE PRODUTOS AVÍCOLAS DO ESTADO DO PARANÁ SINDIAVIAPAR. Avicultura do Paraná. n. 16, 2010. 52 p.
THOMAS, L. Clinical laboratory diagnostics. Frankfurt: THBooks, 1998. 1526 p.

WHIPPLE, G.; KOOHMARAIE, M.; DIKEMAN, M. E.; CROUSE, J. D.; HUNT, M. C.; KLEMM, R. D. Evaluation of attributes that affect longissimus muscle tenderness in Bos taurus and Bos indicus cattle. Journal of Animal Science, Champaing, v. 68, n. 9, p. 2716-2728, 1990. 
